\title{
Mechanism of Subordinate Peak Skewing of FBG Sensor during Cracks Propagation Monitoring on Aluminum Alloy Structure
}

\author{
Bo Jin,, ${ }^{1,2}$ Weifang Zhang, ${ }^{3}$ Feifei Ren, ${ }^{3}$ Meng Zhang, ${ }^{3}$ Wei Dai, ${ }^{3}$ and Yanrong Wang ${ }^{1}$ \\ ${ }^{1}$ School of Energy and Power Engineering, Beihang University, 37 Xueyuan Rd., Haidian District, Beijing 100191, China \\ ${ }^{2}$ Collaborative Innovation Center for Advanced Aero-Engine, Beihang University, 37 Xueyuan Rd., Haidian District, \\ Beijing 100191, China \\ ${ }^{3}$ School of Reliability and Systems Engineering, Beihang University, 37 Xueyuan Rd., Haidian District, Beijing 100191, China \\ Correspondence should be addressed to Wei Dai; dw@buaa.edu.cn
}

Received 7 August 2017; Revised 30 September 2017; Accepted 9 October 2017; Published 20 November 2017

Academic Editor: Lei Yuan

Copyright (C) 2017 Bo Jin et al. This is an open access article distributed under the Creative Commons Attribution License, which permits unrestricted use, distribution, and reproduction in any medium, provided the original work is properly cited.

\begin{abstract}
This study investigates the variety of the spectra features of fiber Bragg grating (FBG) around the crack tip during fatigue crack propagation. The study results reveal that the turning of the subordinate peak is significantly associated with crack lengths and corresponds to strain gradient along the FBG. Meanwhile, the strain distribution sensed by the FBG changes with the sensing section of the grating. FBG sensors could observe the monotonic plastic zone ahead of the fatigue crack tip. The cubic strain is distributed along the grating, with monotonic plastic zone propagation at the initial and terminal part of the grating, at approximately a $30 \%$ ratio of the entire grating. However, the monotonic plastic zone is sensed by the FBG, at $\pm 15 \%$ bias of the grating center, with the quadratic strain gradient pattern along the grating. In particular, when the initial and terminal parts of the grating experience highly inhomogeneous strain distribution, the spectrum distortion occurs.
\end{abstract}

\section{Introduction}

The structural damage of aircraft constructions cannot be avoided over the course of long-term service. Such damage can include fatigue, material aging, corrosion, and cracking problems [1]. Furthermore, structural damage can be identified in synchronous real-time monitoring of the aluminum alloy crack propagation [2]. FBG sensors are recognized and are applicable to structural health monitoring (SHM), such as in the concrete structure of bridges, nuclear power stations, and large dams [3]. This is due to the specific benefits of FBG sensors, such as erosion resistance, small size, and multiplexing [4]. It is argued to be one of the most promising sensors in crack propagation prediction [5].

Several researchers have made contributions to mechanical interpretations of spectral characteristics, changes with crack propagation, and the health monitoring of composite materials. Reference [6] found that the FBG spectrum was sensitive to the delamination propagation behavior of composite materials. The spectrum was split into two peaks dependent on the strain gradient which was caused by edge delamination. The light intensity of the primary peak increases with the extent of edge delamination. In addition, further interpretation of spectral features changed with the extension of delamination [7]. Regarding predictions of delamination size in composites, an indicator of the light intensity ratio of the primary peak to the subordinate peak was proposed by [8]. In addition, [9] states that the two peaks at lower and higher wavelengths in the reflection spectra correspond to the various forms of strain distribution, with the increment of delaminated area of composites.

Regarding strain distribution analysis around the crack tip, many numerical approaches are developed to investigate the strain distribution, such as FEM and the LocalGlobal method. Reference [10] states that the plastic zone is divided into three regions: the monotonic plastic zone, the cyclic plastic zone, and the process zone. Meanwhile, several experimental advancements have already improved the strain distribution calculation of aluminum, such as digital image correlation (DIC), the laser speckle method, and laser speckle imaging (LSI). These improvements are also accompanied by increased instrument precision and accuracy. Strain sensing 
sensors include eddy current measurement sensors, strain gauge, and the coupling fiber-optic strain sensor [11] for the strain sensitivity of FBG. The latter is used for strain monitoring; however, it is costly and is used for the monitoring of structural deformation. Therefore, the method is unsuitable for the local stress concentration, such as the strain concentration around the crack tip. Consequently, the FBG is considered as a potential sensor for the monitoring of aluminum crack concentration.

However, there has been little research focused on the mechanism of subordinate peak skewing of the FBG on aluminum crack propagation. Peters et al. [12] discussed the spectra of the FBG as a possible basis for the resolution of an arbitrary applied strain distribution. Jin et al. [13] discussed the subordinate peak of the reflection spectrum in crack propagation monitoring of aluminum alloys. The FBG was placed parallel to the external loading direction. However, in this paper, the FBG was applied perpendicular to the axial direction of the external load in this study. Subordinate peak variation which shows a strong correlation to the strain region size with crack propagation was demonstrated in this research.

\section{Experiment}

The core principle was to investigate the mechanism on various physical characteristics, extracted from the spectrum intensity during crack propagation. The mechanism of subordinate wavelength variation during the crack propagation demonstrated practical significance regarding quantifiable crack identification. Thus, the structure is equipped with sensors and interacts with the crack propagation, which presents the evolution of the state and physical parameters.

2.1. Specimen. The objective system established in this paper is a plate made of aluminum alloy 7075-T6, with the dimension of $300 \mathrm{~mm} \times 100 \mathrm{~mm} \times 2 \mathrm{~mm}$ as shown in Figure 1 . A $10 \mathrm{~mm}$ diameter hole was drilled into the center of the plate. A $3 \mathrm{~mm}$ precrack is introduced by electrical discharge machining to activate fatigue crack propagation. The top frontier is fastened and a uniform tensile load of $65 \mathrm{MPa}$ is implemented from the bottom, as indicated in Figure 1. Table 1 shows specimen properties. To sense the strain in various shapes distributed along the grating caused by the crack tip, the FBG sensor is adhered parallel to the precrack direction. The horizontal distance from the initial grating to the hole edge is $7 \mathrm{~mm}$, and the perpendicular distance to the horizontal line is $1 \mathrm{~mm}$. The FBG sensor length is $10.1 \mathrm{~mm}$. A liquid cyanoacrylate adhesive is used to glue the FBG sensor onto the specimen surface. Young's modulus of the adhesive is $1.75 \mathrm{MPa}$.

2.2. Experiment Setup. A fatigue crack damage recognition test platform is developed and FBG sensors are utilized to abstract the damage indicator. Figure 2 illustrates the hole-edge crack experimental setup which is composed of three main segments: fatigue loading equipment, the optical modulation analyzer, and a fatigue crack detection device.
TABLE 1: Mechanical properties of 7075-T6 aluminum alloy.

\begin{tabular}{lcccc}
\hline Material & $\begin{array}{c}\text { Tensile } \\
\text { strength } \\
(\mathrm{MPa})\end{array}$ & $\begin{array}{c}\text { Yield } \\
\text { strength } \\
(\mathrm{MPa})\end{array}$ & $\begin{array}{c}\text { Poisson's } \\
\text { ratio } \\
(\mathrm{MPa})\end{array}$ & $\begin{array}{c}\text { Elastic } \\
\text { modulus } \\
(\mathrm{MPa})\end{array}$ \\
\hline $\begin{array}{l}\text { AL7075- } \\
\text { T6 }\end{array}$ & 572 & 503 & 0.33 & 73100 \\
\hline
\end{tabular}

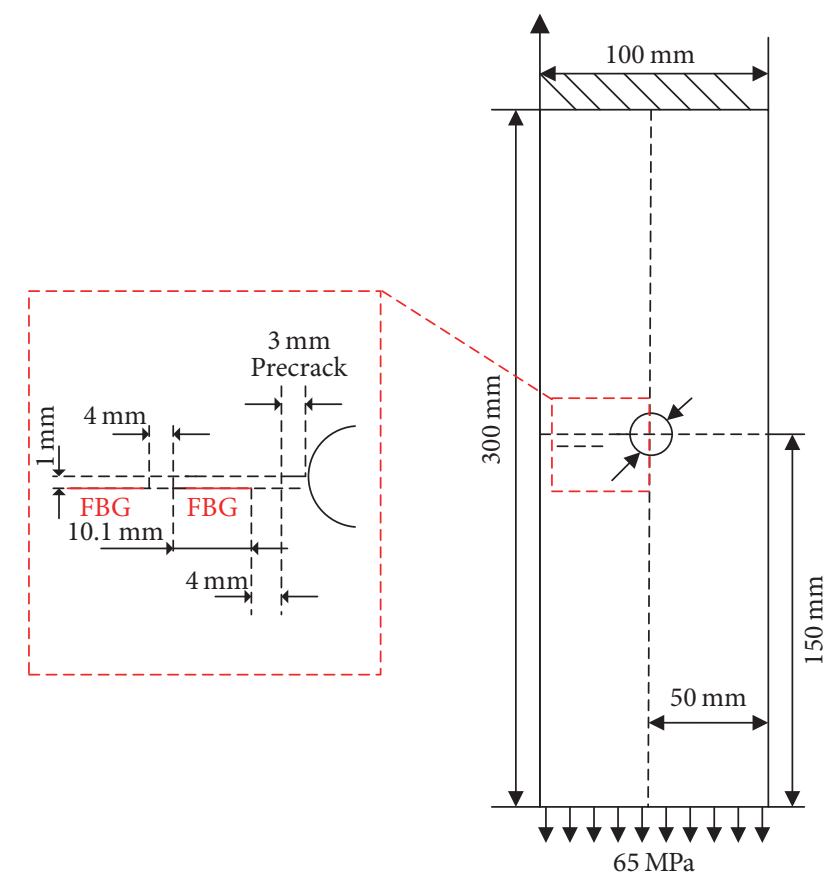

FIGURE 1: Schematic of the aluminum specimen.

The reflected spectrum of the FBG is obtained by an optical demodulator (SM125, Micro Optics Inc.), which possesses high wavelength accuracy. An optical microscope oversees the fatigue crack propagation with a Charge Coupled Device (CCD) camera during the loading progression. Fatigue testing is undertaken using a hydraulic MTS machine with continuous fatigue loading along the axial direction as shown in Figure 2. The spectra data and crack length were collected with the increase of crack length of about $1.0 \mathrm{~mm}$ visually, in which the fatigue loading machine was shut down and modulated at the maximum load value. The constant amplitude loading spectra used in this study have a maximum value of loading set at $65 \mathrm{MPa}$ and a cycling frequency of $10 \mathrm{~Hz}$. The FBG sensor number is FSSR5025.

\section{Results}

The natural crack in the plate was initiated and propagated through the use of a cyclic fatigue test. The FBG sensor was used to measure strain distribution along the axial grating at differential strain profiles with the crack propagation. The data was obtained using the FBG sensing demodulation system throughout cyclic fatigue. Initially, the reflection spectra are symmetrical and had a primary reflection spectrum. Subsequently, the peak simply moves backwards or 


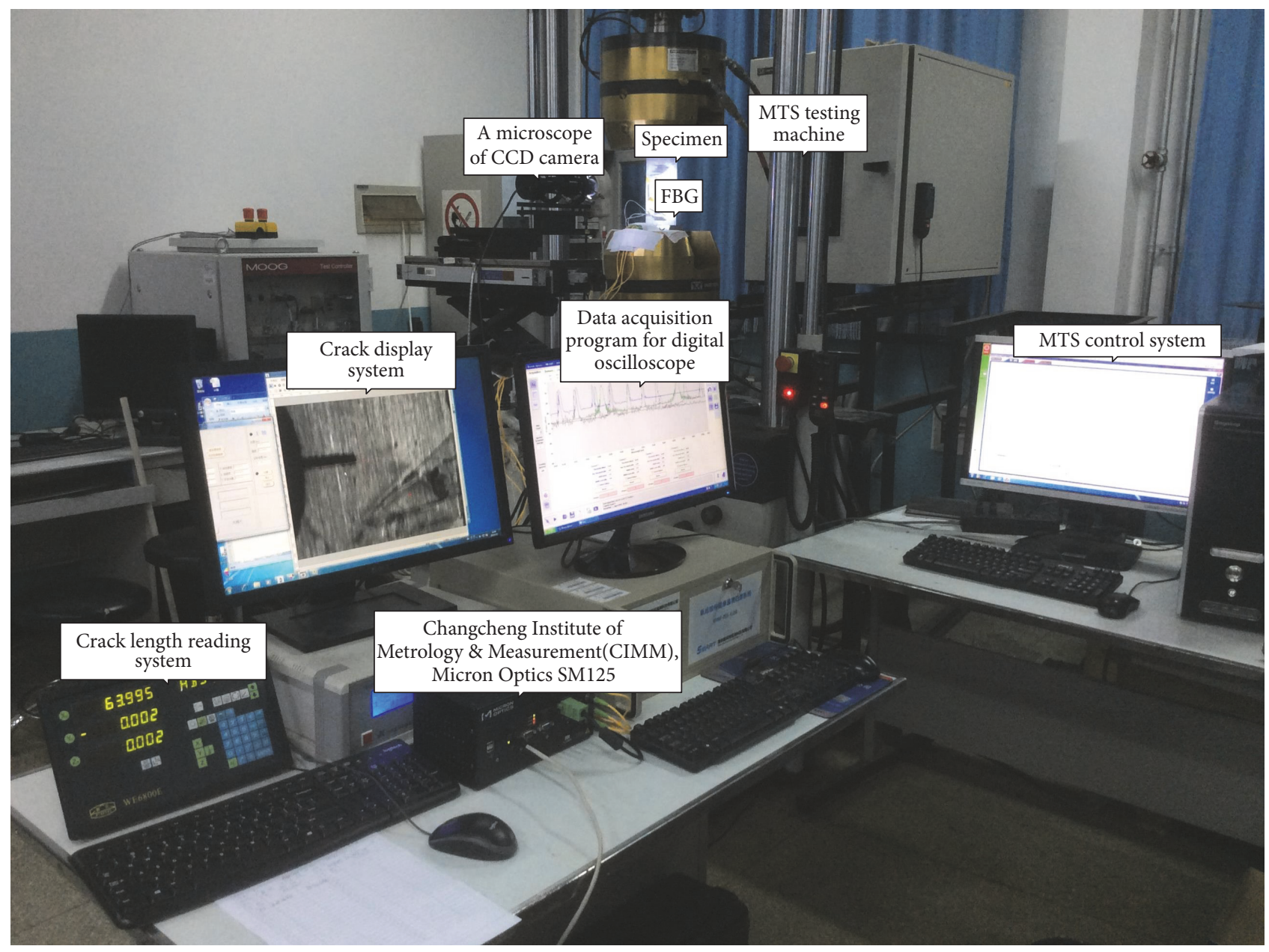

FIGURE 2: Experiment setup for the crack detection in aluminum structure.

forwards when the grating sensed uniformity strain profile. Concurrently, the pitch changes accordingly with the internal periodic modulation and all grating periods experience changes synchronously. This results in a shift of the Bragg wavelength without modification of the spectrum shape, and consequently the spectrum becomes symmetric and smooth due to the uniformity of the strain distribution along the grating (Figure 3). However, regarding crack propagation, the spectral wavelength shifts gradually and the reflection spectrum showed a significant change in shape. This is represented as subordinate peaks as shown in Figures 5(a), 6(a), and 7(a). Meanwhile, the FWHM also displayed an obvious correlation with the crack length until a saturation crack length is reached. The spectrum bandwidth is also directly related to strain distribution and hence has been previously applied $[14,15]$. A shift of the measured central wavelength of less than $0.1 \mathrm{~nm}$ is observed during the process. The reason is that there is a small offset between the center of the FBG and the intersection point between the FBG and the neutral line.

It is clearly demonstrated that the strain patterns are distributed around the crack tip according to perfect nonuniformity. Thus, nonuniform strain is distributed along the entire length of the FBG with the crack propagation. Meanwhile, the subordinate peaks vary with the complex strain distribution along the grating. In addition, the relationship between the subordinate peak and the strain distribution pattern has been discussed and simulated in previous research [13]. The turning subordination peaks coincide with the crack length, which is represented in the comparison between Figures 5(a) and 6(a) with Figure 7(a). The presence of crack propagation introduced three regions (Zones $\mathrm{A}, \mathrm{B}$, and $\mathrm{C}$ ) of uniform grating strain, creating two primary peaks in the reflected spectrum as shown in Figure 4.

The distribution of strain patterns along the grating is mainly due to the ratio of crack length lying in the grating with the entire grating (Figure 4). Additionally, the crack propagation rate is a major factor in determining the monotonic plastic zone propagation rate. Regarding the cracks, the propagation rate affects the ratio of crack length lying in the grating with the entire grating. Reference [16] shows that crack propagation rate changes with external applied stress and this ratio could be changed with external loading stress.

And when the crack approaches initial grating, approximately $30 \%$ of the entire grating ratio, positioning at $-0.2-3.2 \mathrm{~mm}$ to initial grating (Zone A), the reflection spectra appear to have subordinate peaks located to the left side of the primary peak. However, the subordinate peak appears at 


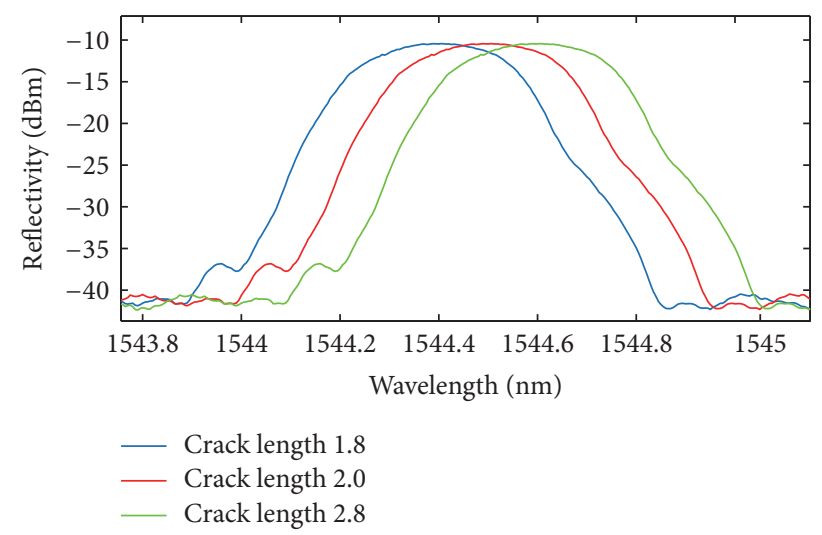

FIGURE 3: Reflection spectrum of FBG in uniform strain distribution.

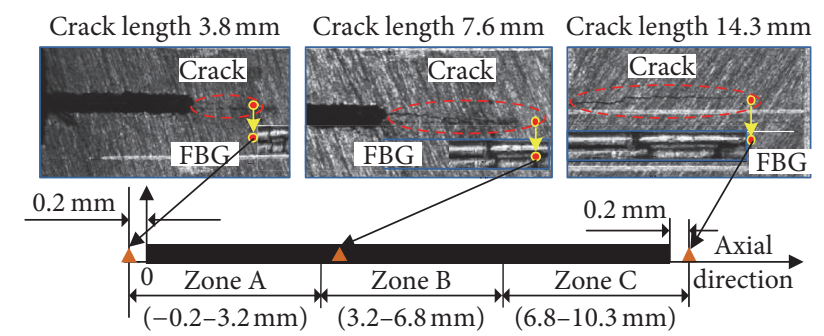

FIGURE 4: Three sensing regions of the grating.

higher wavelengths when the crack approaches $\pm 15 \%$ bias of the central grating, positioned at $3.2-6.8 \mathrm{~mm}$ to the initial grating (Zone $\mathrm{B}$ ). When the crack propagates to the terminal grating, at approximately $30 \%$ ratio of the entire grating, positioning at $6.8-10.3 \mathrm{~mm}$ to initial grating (Zone C), the reflection spectra appear to have subordinate peaks located to the left side of the major peak.

3.1. Analysis of Spectra in Zone A Sensing Grating. When the crack propagates close to observation Zone A, the subordinate peak is initially presented in the lower wavelength and drifts towards the same orientation. Moreover, the peak broadens and the subordinate peak energy increases as shown in Figure 5(a). Typically, the reflected spectra broaden and multiple peaks appear. The spectrum modifications may be due to polarization-induced lateral strains and/or nonhomogeneous strains parallel to the fiber axis.

If the FBG is submitted to different strain patterns along the grating, different lower peaks distort the reflected peak, as shown by curve A in Figure 5(a), and more details are shown in Figure 5(b). The slitting of the spectra occurred and the spectra shape was distorted. Afterwards, the spectra enlarged and the spectra distortion is critical, which corresponds to the outcomes by Ussorio [17]. The reflection spectra are evenly slit into several peaks at the initial and eventual break of the grating. The form of reflection spectra depends on the strain distribution along the grating [18]. Thus, the serve nonuniform strain pattern along the grating is assumed to be the principal reason for the spectra oscillation [19].
Figure 5(a) also shows a spectral wavelength shift towards a lower wavelength, with crack propagation in Zone A. These indicate a decrease of tensile strain in the grating. Thus, the central wavelengths diminished from $1544.7 \mathrm{~nm}$ to $1544.6 \mathrm{~nm}$.

The deformity of the reflection spectra is generally related to the strain distribution along the FBGs [20]. In fact, the distribution of strain patterns along the grating corresponds to crack propagation. Regarding crack propagation, the spectrum bandwidth and full width at half maximum (FWHM) [21] broadened, accompanied by a sudden broadening of the spectrum that occurs at a $5.4 \mathrm{~mm}$ crack length, in comparison with curve A in Figure 5(a). Meanwhile, the bandwidth of FWHM broadens from $0.3 \mathrm{~nm}$ to $0.4 \mathrm{~nm}$, and the FWHM increases from $0.3 \mathrm{~nm}$ to $0.7 \mathrm{~nm}$, with the crack propagating in Zone A.

3.2. Analysis of Spectra in Zone B Sensing Grating. As the crack propagates to Zone B with a crack length of $7.6 \mathrm{~mm}$, the number of subordinate peaks increases, as shown in Figure 6(b). Meanwhile, the reflection spectra appear to have multiple peaks located at the right side of the primary peak. Figure 6(a) also shows that the spectral intensity shifts towards a lower wavelength, with the crack propagation in Zone B. This indicates a decrease in tensile strain at the grating. Thus, the central wavelengths diminish from $1544.2 \mathrm{~nm}$ to $1544.1 \mathrm{~nm}$.

The crack in the aluminum material can produce a strain concentration or gradient zone, which contains complex strain patterns. If differential strain patterns are applied to the grating, it will suffer from a nonuniform strain distribution pattern. This causes a sensor response that is more complicated than uniform cases $[22,23]$. The nonuniform strain along the grating will change the periodicity of the grating pattern. The grating pattern is modified from a uniform to a chirped configuration [24], as shown in Figure 6(a). Thus, the width of FWHM broadened from $0.6 \mathrm{~nm}$ to $1.0 \mathrm{~nm}$. Simultaneously, longer wavelength subordinate peaks appear and drift to naturally similar orientations.

3.3. Analysis of Spectra in Zone C Sensing Grating. For crack propagation towards Zone $\mathrm{C}$ terminal with a crack length of $14.3 \mathrm{~mm}$, the distortion of the spectrum occurs as shown in Figure 7(b). Meanwhile, the reflection spectra are slit into multiple peaks located on the left side of the primary peak. Thus, it is confirmed that the spectrum oscillation can be related to the nonuniform strain distribution along the grating caused by transverse crack.

Figure 7(a) also shows that the spectral intensity shifts towards a lower wavelength, with crack propagation in Zone C. Thus, the central wavelength diminishes from $1544.7 \mathrm{~nm}$ to $1544.6 \mathrm{~nm}$, and the width of FWHM broadens from $0.5 \mathrm{~nm}$ to $1.0 \mathrm{~nm}$.

\section{Discussion}

4.1. Mechanism of the Skewing of the Subordinate Wavelength. Generally, when the compressional strain is sensed by the 


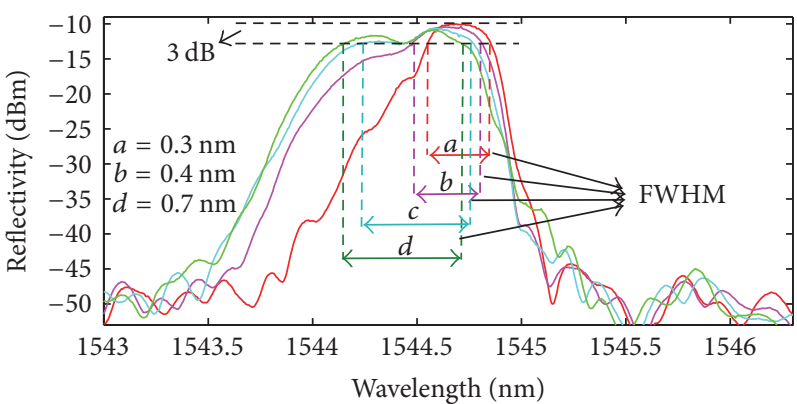

- $a$ crack length 3.8 - $c$ crack length 5.4 - $d$ crack length 6.9

(a)

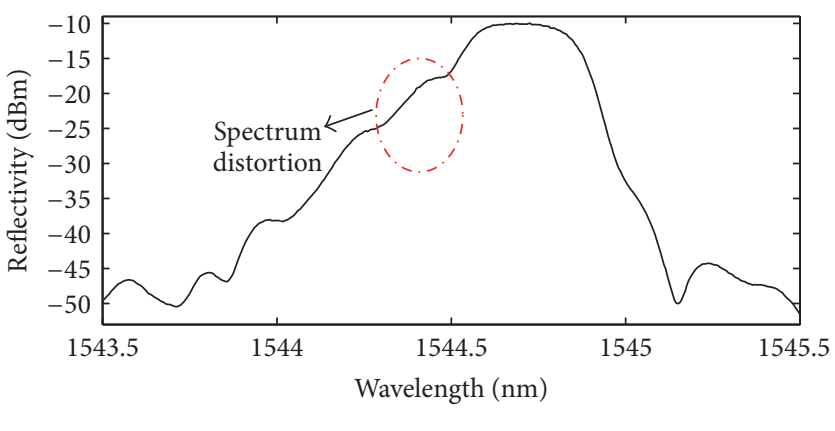

_ Crack length 3.8

FIgURE 5: Reflection spectra of FBG in Zone A sensing grating. (a) The global curves; (b) the distortion of spectrum.

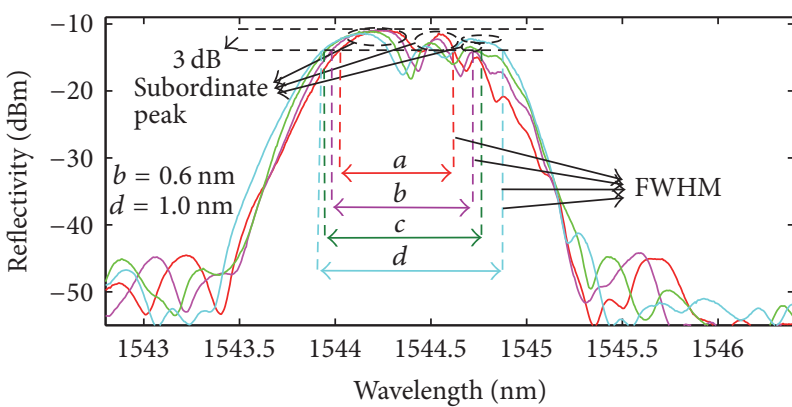

$a$ crack length 7.6
$-b$ crack length 8.6

- $c$ crack length 10.2

$d$ crack length 10.8

(a)

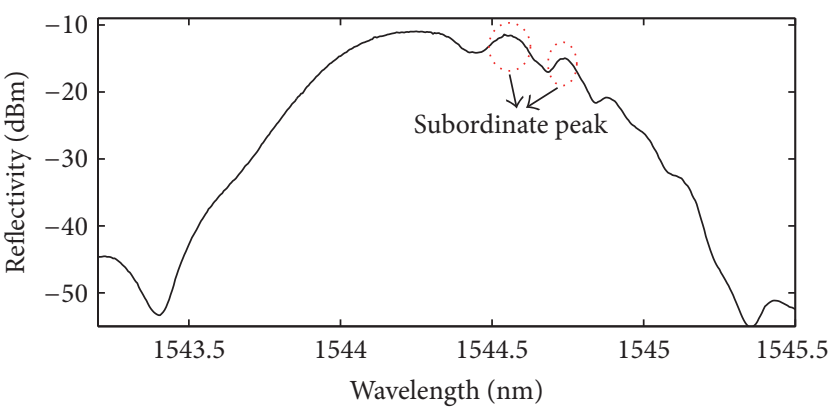

— Crack length 7.6

FIgURE 6: Reflection spectra of FBG in Zone B sensing grating. (a) The global curve; (b) subordinate peaks of the intensity spectra.

FBG, the reflection spectra move towards a lower wavelength. While tensile strain is sensed by FBG, the reflection spectra will shift to a higher wavelength [25]. There is a high order of the strain distribution around the crack of the test specimen. Thus, the strain distribution along the grating is nonuniform and the width of the spectra broadens. As the FWHM of reflection spectra increases, reflectance decreases and the subordinate peaks appear initially, and even spectra distortion occurs.

Reference [13] investigates the subordinate peak located in the lower wavelength, with quadratic strain distribution being employed along the grating of the fiber. When the cubic strain gradient is employed along the grating of the fiber, the subordinate peak is located at a higher wavelength. Meanwhile, the strain distribution along the grating corresponds to the plastic zone ratio ahead of the crack tip to the entire grating. When the crack propagates to the initial or terminal part of grating, at approximately $30 \%$ ratio of the entire grating, the cubic strain is distributed along the grating. However, the monotonic plastic zone is sensed by the FBG, at $\pm 15 \%$ bias of the grating center, with the quadratic strain gradient pattern along the grating.

Results conclude that the FWHM and location skewing of the subordinate peak are related to the change of strain distribution along the grating, with the crack propagation. The FWHM and the subordinate peak skewing are proved to be good indicators for real-time crack propagation evaluation.

4.2. Analysis of the Spectrum Distortion. Crack progression causes a nonuniform strain field around the crack tip to reach the grating area. This modifies the FBG response by significant variations with the subordinate reflected peak. As previously discussed, a severe strain distribution pattern, such as linear, quadratic, and cubic strain distributions, is sensed by the grating and can be attributed to the complex strain distribution around the crack tip. Previous research reveals that the linear and quadratic strain can be perceived by the FBG, which can be obtained by experiment and simulation. Meanwhile, Peters et al. [26] stated that the cubic strain gradient can be perceived by FBG, through the development of an experiment to calibrate the strain distribution pattern along the grating.

Meanwhile, [27] proposes that axial and transverse strain can both be sensed by the FBG, especially when the grating is adhered perpendicular to the loading direction. However, it is still a challenge to identify whether the distortion is promoted 


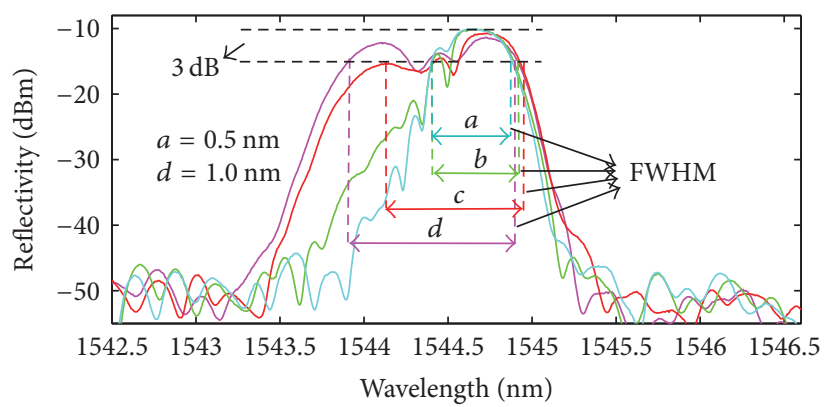

$\begin{array}{ll}a \text { crack length } 11.2 & \quad c \text { crack length } 13.6 \\ -b \text { crack length } 11.6 & \quad d \text { crack length } 14.3\end{array}$

(a)

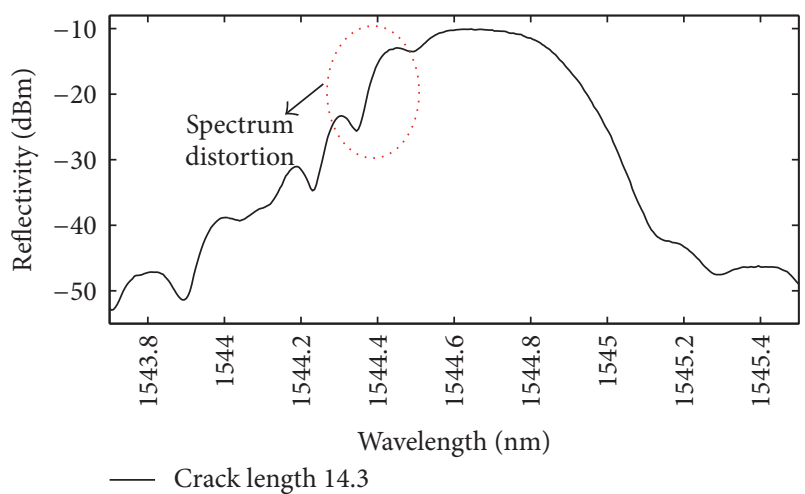

(b)

FIGURE 7: Reflection spectra of FBG in Zone C sensing grating. (a) The global curve; (b) the distortion of spectrum.

by a nonuniform longitudinal strain or by transverse stress splitting.

Thus, it is concluded that the complex strain distribution pattern sensed by FBG could be the major reason of spectrum distortion, which correlates with the distance of crack tip from the initial grating.

\section{Conclusion}

To determine the presence and development of the crack, FBGs show significant changes in the strain due to crack propagation in the specimen. In this study, the experimental data from fatigue testing is used to analyze the characteristic parameters for different crack lengths, in combination with the variable strain distribution. The spectrum became deformed with crack propagation, such as the number of subordinate peaks, and subordinate peak skewing. The change of strain distribution pattern sensed by the grating is assumed to cause the subordinate peak skewing, with the crack propagation. The monotonic plastic zone ahead of the crack tip is sensed by the grating, and the strain distribution pattern is closely related to ratio of sensed subgrating to the entire grating. When the crack propagates to approximately $30 \%$ of the initial or terminal grating, the cubic strain distribution is sensed by the FBG.

Furthermore, the FBG-based demodulation method can be used as a novel and alternative technique to real-time detection of the transverse cracks within the complex structure of aluminum alloy.

\section{Conflicts of Interest}

The authors declare no conflicts of interest.

\section{Authors' Contributions}

Bo Jin and Wei Dai conceived the key idea and designed the experiments. Weifang Zhang provided the academic support and checked the manuscript. Feifei Ren and Meng Zhang performed the experiment. And Yanrong Wang contributed to optical measurement tools. All authors made contributions to the writing and revising of the manuscript.

\section{Acknowledgments}

This work was supported by the Technical Foundation Program (Grant no. KG06002201) from the Ministry of Industry and Information Technology of China.

\section{References}

[1] D. Kinet, P. Mégret, K. W. Goossen, L. Qiu, D. Heider, and C. Caucheteur, "Fiber bragg grating sensors toward structural health monitoring in composite materials: challenges and solutions," Sensors, vol. 14, no. 4, pp. 7394-7419, 2014.

[2] J. P. Ou, "Some recent advance of intelligent monitoring system for civil infrastructures in mainland China," in Proceedings of the First International Conference on Structural Health Monitoring and Intelligent Infrastructures, pp. 131-144, Tokyo, Japan, 2003.

[3] K. Manfred, Strain Measurement with Fiber Bragg Grating Sensors, HBM, 2006.

[4] S. Cieszczyk and P. Kisała, "Inverse problem of determining periodic surface profile oscillation defects of steel materials with a fiber Bragg grating sensor," Applied Optics, vol. 55, no. 6, pp. 1412-1420, 2016.

[5] L. C. Hollaway, "A review of the present and future utilisation of FRP composites in the civil infrastructure with reference to their important in-service properties," Construction and Building Materials, vol. 24, no. 12, pp. 2419-2445, 2010.

[6] N. Takeda, Y. Okabe, and T. Mizutani, "Damage detection in composites using optical fibre sensors," in Proceedings of the Institution of Mechanical Engineers Part G Journal of Aerospace Engineering, vol. 221, pp. 497-508.

[7] S. Takeda, Y. Okabe, and N. Takeda, "Monitoring of delamination growth in CFRP laminates using chirped FBG sensors," Journal of Intelligent Material Systems and Structures, vol. 19, no. 4, pp. 437-444, 2008.

[8] N. Takeda, Y. Okabe, J. Kuwahara, S. Kojima, and T. Ogisu, "Development of smart composite structures with smalldiameter fiber Bragg grating sensors for damage detection: 
quantitative evaluation of delamination length in CFRP laminates using Lamb wave sensing," Composites Science and Technology, vol. 65, no. 15-16, pp. 2575-2587, 2005.

[9] R. Ramly, W. Kuntjoro, and M. K. Abd Rahman, "Embedded FBG sensor in aircraft smart composite materials for structural monitoring," Applied Mechanics and Materials, vol. 393, pp. 311316, 2013.

[10] K. M. Lal, "On the evaluation of monotonic and cyclic plastic zones," Engineering Fracture Mechanics, vol. 9, no. 2, pp. 433442, 1977.

[11] B. Ma, "Research of coupling single-mode fiber-optic vibration sensor and experimental test," Applied Mechanics and Materials, vol. 405-408, pp. 3318-3322, 2013.

[12] K. Peters, P. Pattis, J. Botsis, and P. Giaccari, "Experimental verification of response of embedded optical fiber Bragg grating sensors in non-homogeneous strain fields," Optics and Lasers in Engineering, vol. 33, no. 2, pp. 107-119, 2000.

[13] B. Jin, W. Zhang, M. Zhang, F. Ren, W. Dai, and Y. Wang, "Investigation on characteristic variation of the FBG spectrum with crack propagation in aluminum plate structures," Materials, vol. 10, no. 6, p. 588, 2017.

[14] Y. Okabe, S. Yashiro, T. Kosaka, and N. Takeda, "Detection of transverse cracks in CFRP composites using embedded fiber Bragg grating sensors," Smart Materials and Structures, vol. 9, no. 6, pp. 832-838, 2000.

[15] P. Childs, A. C. L. Wong, W. Terry, and G. D. Peng, "Measurement of crack formation in concrete using embedded optical fibre sensors and differential strain analysis," Measurement Science and Technology, vol. 19, no. 6, Article ID 065301, 2008.

[16] G. Forman Royce and J. P. Hudson, "Digital computer program for the analysis of crack propagation in cyclic loaded structures," 1967.

[17] M. Ussorio, "Modifications to FBG sensor spectra due to matrix cracking in a GFRP composite," Construction and Building Materials, vol. 1, pp. 111-118, 2006.

[18] R. Di Sante, "Fibre optic sensors for structural health monitoring of aircraft composite structures: recent advances and applications," Sensors, vol. 15, no. 8, pp. 18666-18713, 2015.

[19] K. Peters, M. Studer, J. Botsis, A. Iocco, H. Limberger, and R. Salathé, "Embeded optical fiber bragg grating sensor in a nonuniform strain field: measurements and simulations," Experimental Mechanics, vol. 41, no. 1, pp. 19-28, 2001.

[20] S. Huang, M. Leblanc, M. M. Ohn, and R. M. Measures, "Bragg intragrating structural sensing," Applied Optics, vol. 34, no. 22, pp. 5003-5009, 1995.

[21] H. Ahmad, M. Z. Zulkifli, K. Thambiratnam, S. F. Latif, and S. W. Harun, "High power and compact switchable bismuth based multiwavelength fiber laser," Laser Physics Letters, vol. 6, no. 5, pp. 380-383, 2009.

[22] J. Zhou, Z. Zhou, and D. Zhang, "Study on strain transfer characteristics of fiber bragg grating sensors," Journal of Intelligent Material Systems and Structures, vol. 21, no. 11, pp. 1117-1122, 2010.

[23] J. F. Botero-Cadavid, J. D. Causado-Buelvas, and P. Torres, "Spectral properties of locally pressed fiber bragg gratings written in polarization maintaining fibers," American Institute of Physics, pp. 248-253, 2008.

[24] O. Andreas and K. Kalli, "Fiber bragg gratings: fundamentals and applications in telecommunications and sensing," APS Meeting Abstracts, p. 61, 2004.
[25] K. Peters, "Networking of optical fiber sensors for extreme environments," in Proceedings of the Sensors and Smart Structures Technologies for Civil, Mechanical, and Aerospace Systems 2016, usa, March 2016.

[26] K. J. Peters, M. Studer, J. Botsis et al., "Measurement of stress concentrations using embedded optical fiber Bragg grating sensors," in Proceedings of the 1999 Symposium on Smart Structures and Materials, pp. 195-206, Newport Beach, Calif, USA.

[27] D. Balageas, Structural Health and Monitoring, Great Britain, Antony power Ltd, London, UK. 


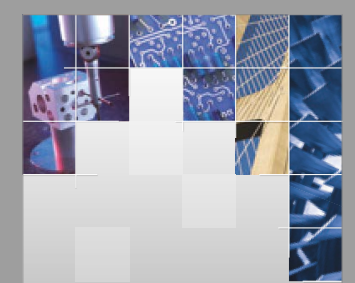

\section{Enfincering}
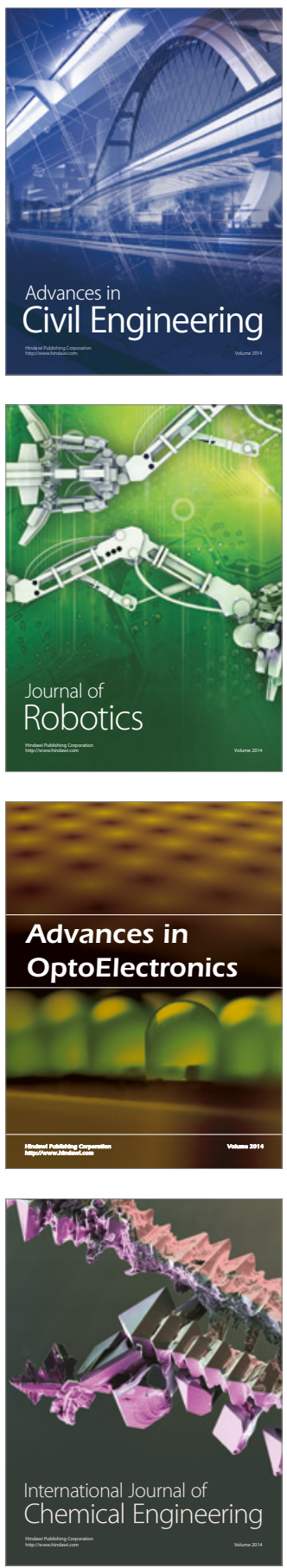

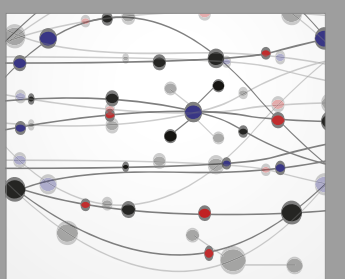

The Scientific World Journal

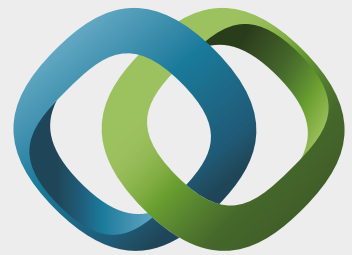

\section{Hindawi}

Submit your manuscripts at

https://www.hindawi.com
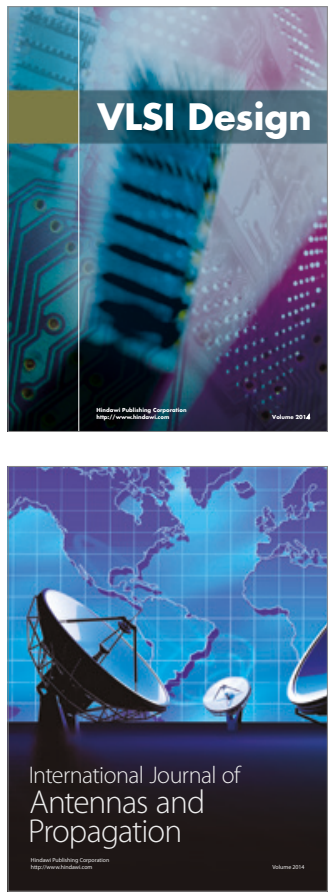

\section{Rotating}

Machinery
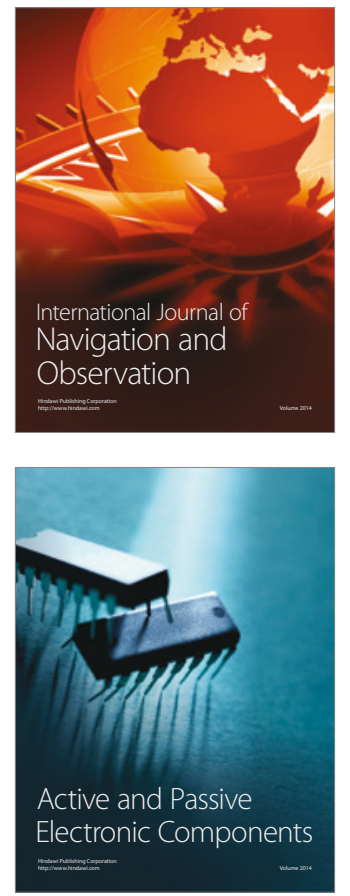
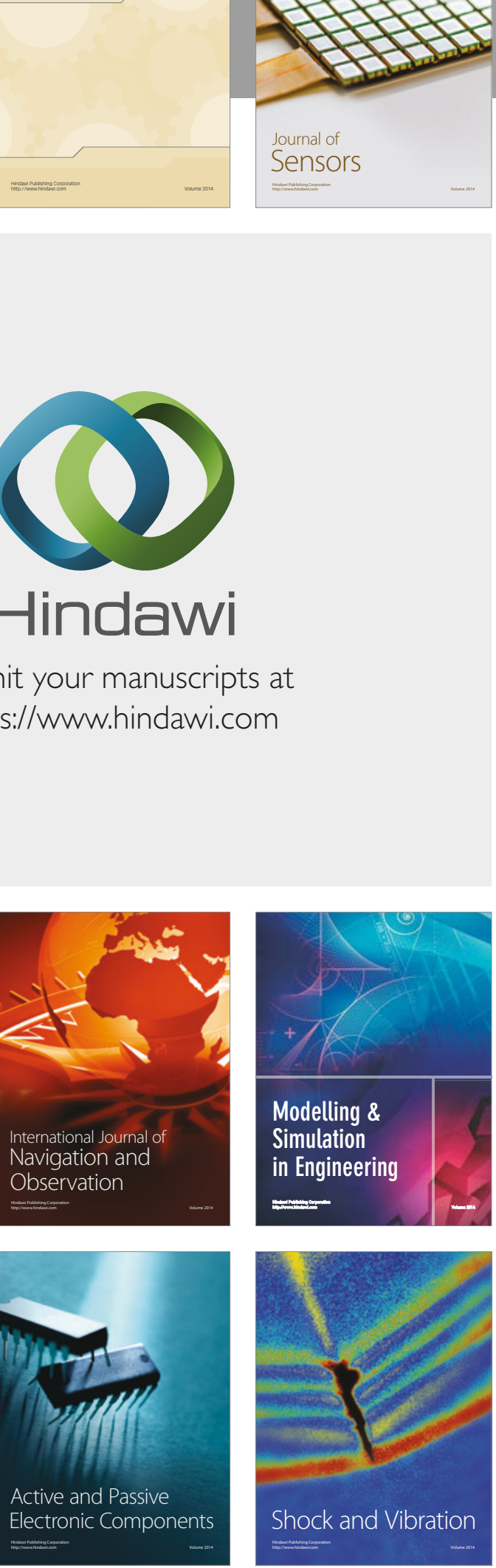
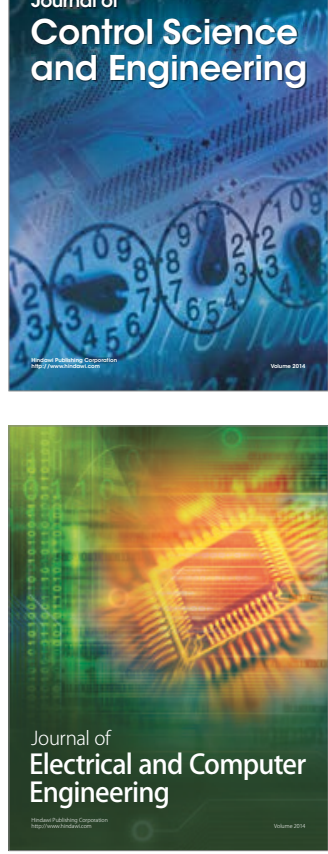

Distributed

Journal of

Control Science

and Engineering
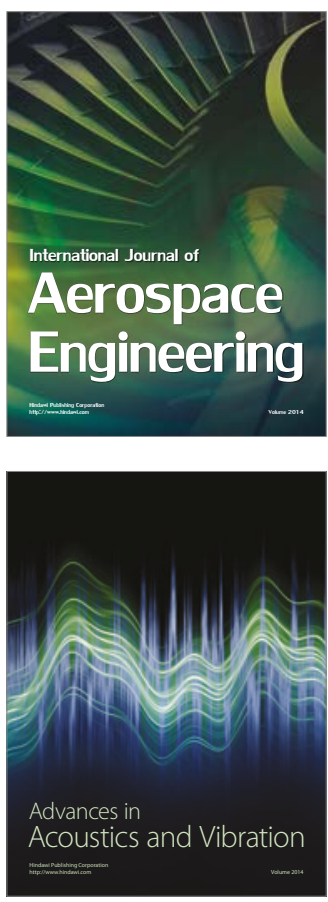

Sensor Networks 